\title{
Os efeitos da estimulação elétrica funcional na marcha de crianças com paralisia cerebral hemiparética após estimulação dos músculos tibial anterior e tríceps sural The effects of functional electrical stimulation on gait of children with hemiparetic cerebral palsy after stimulation of the anterior tibial and triceps surae muscles
}

Fabiene Balbino Lima da Costa*, Laila Cristina Moreira Damázio, M.Sc.**, Fernanda Granja Ferreira Melo, M.Sc.***

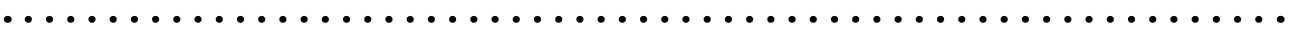

*Graduada em Fisioterapia pela Faculdade Regional de UbálMG - Universidade Presidente Antônio Carlos Campus II,

**Docente do curso de fisioterapia da Unipac/Ubá, Especialista em fisioterapia Neurológica e PSF,

***Docente do curso de fisioterapia da Unipac/Ubá, Especialista em Biomecânica pela UFRJ

\section{Resumo}

A paralisia cerebral (PC) interfere na execução dos movimentos devido aos padrôes anormais da coordenação, postura e tônus muscular. Estima-se que no Brasil existam 17 mil novos casos por ano, sendo considerado problema social e de saúde pública. A hemiparesia se caracteriza por espasticidade no hemicorpo contralateral ao hemisfério cerebral afetado. A marcha hemiparética não apresenta o toque do calcâneo no início do apoio bipodal pela incapacidade do músculo tibial anterior em produzir tensão suficiente para realizar o movimento de dorsiflexão. A fase de impulsão também fica comprometida em decorrência da fraqueza do músculo tríceps sural, constituindo fator limitante para a marcha. Estudos morfológicos sugerem que a fraqueza do músculo parético é mais incapacitante que a espasticidade, ela ocorre devido ao desuso, perda de unidades motoras, alteração na ordem do recrutamento, tornando-se necessário o fortalecimento desta musculatura para melhorar a capacidade de geraçáo de força e habilidade motora. A estimulaçáo elétrica funcional (FES) promove fortalecimento com possibilidade de redução da assimetria cortical, pois envolve processos relacionados à movimentação voluntária. O objetivo do presente estudo é comparar os efeitos da FES na funcionalidade da marcha em pacientes com PC após sua aplicação nos músculos tibial anterior e tríceps sural. Os resultados obtidos pela escala GMFM constataram maior evolução no paciente que recebeu a FES no músculo tríceps sural, assim como melhora da ADM passiva e aumento de força muscular de dorsiflexores e plantiflexores, redução do grau de espasticidade e evolução nas variáveis da marcha. Desta forma a FES demonstrou resultados positivos no tratamento de musculatura espástica com evolução na funcionalidade da marcha.

Palavras-chave: paralisia cerebral, estimulação elétrica funcional, hemiparesia, espasticidade.

\begin{abstract}
The cerebral palsy (CP) acts in the implementation of the movements due to abnormal patterns of coordination, posture and muscle tone. It is estimated that in Brazil there are 17,000 new cases per year, which is considered a social and public health problem. The hemiparesis is characterized by spastic paralysis in the contralateral half part of the body of the affected cerebral hemisphere. The hemiparetic gait does not show the touch of the calcaneus at the beginning of the bipedal support because the tibialis anterior muscle does not produce enough strength to perform dorsiflexion movements. The push to walk is also damaged as result of the weakness of the surae triceps muscle, which is a limiting factor for gait. Morphological studies suggest that the weakness of the paretic muscle is more disabling than the spasticity, and occurs due to disuse, motor unit loss and changes in recruitment order. In order to improve strength and motor skill, the muscles should be strengthened. The Functional Electrical Stimulation (FES) promotes improvement on strength and may reduce cortical asymmetry, as it involves voluntary body movement processes. The objective of this study was to compare the effects of FES on gait function in patients with $\mathrm{CP}$ after using it in the tibialis anterior and triceps surae muscles. The results obtained by the GMFM score showed a great improvement of patients who received FES in the surae triceps muscle, as well as improvement in the passive range of motion of muscle strength in dorsiflexors and plantiflexors, reducing the degree of spasticity and changes in gait variables. The FES showed positive results in treating spastic muscles and improvements in gait function.
\end{abstract}

Key-word: cerebral palsy, functional electrical stimulation, hemiparesis, spasticity. 


\section{Introdução}

A paralisia cerebral (PC) é uma doença não progressiva de múltiplas etiologias, que resultam em lesão do sistema nervoso central, em estágios iniciais do desenvolvimento do encéfalo. Constitui uma das mais importantes doenças neurológicas referidas, interfere na execução dos movimentos em decorrência dos padróes anormais da coordenaçáo, postura e tônus muscular, dificultando ou impedindo a aquisição das habilidades motoras e cognitivas $[1,2]$.

Sua incidência em países desenvolvidos é de 2:1000 nascimentos, em países subdesenvolvidos é de 7:1000 nascimentos. No Brasil dados estimam cerca de 17 mil novos casos por ano, sendo considerada problema social e de saúde pública [3,4].

Pode ser classificada em espástica, atetóide, atáxica, hipotônica ou mista. Sendo que a forma espástica é a mais comum e pode ser classificada de acordo com a topografia em quadriplegia/quadriparesia, diplegia/diparesia ou hemiplegia/ hemiparesia [2]. A hemiparesia se caracteriza por déficit motor com espasticidade unilateral, atingindo o dimídio contralateral ao hemisfério cerebral afetado [5].

A espasticidade é consequência de distúrbios dos reflexos espinhais proprioceptivos, manifesta-se clinicamente por movimento abrupto de hiperreflexia do tendão ao movimento passivo e co-contração muscular agonista-antagonista. As adaptaçóes secundárias à espasticidade constituem fraqueza e atrofia muscular, alteraçóes dos tecidos conectivos e diminuição da qualidade da função motora, produzindo incapacidades consideráveis das atividades de vida diária [6].

A marcha hemiparética apresenta extensão do membro inferior e pé equinovaro no dimídio acometido, consequentemente não há o toque do calcâneo no início do apoio bipodal, pela incapacidade do músculo tibial anterior em produzir tensão suficiente para realizar o movimento de dorsiflexão. A fase de impulsão também fica comprometida em decorrência da fraqueza do músculo tríceps sural, o músculo mais importante na execução da flexão plantar. Este fator é limitante para a transferência de sentado para de pé, a permanência em posição ortostática e marcha [6].

Alguns estudos morfológicos dos músculos paréticos sugerem que a fraqueza muscular é mais incapacitante que a espasticidade, esta ocorre em consequência do desuso, da perda dos efeitos tróficos centrais, da atrofia neurogênica, da perda das unidades motoras e da alteração na ordem do recrutamento. Desta forma o fortalecimento é necessário para melhorar a eficiência, a capacidade da geração de força e a habilidade motora $[3,6]$.

O aumento da força por meio de programa de fortalecimento nos membros inferiores paréticos está associado com a melhora da velocidade e cadência da marcha, execução de tarefas funcionais como caminhar, sentar, levantar, subir e descer escadas, além do aumento do equilíbrio durante a fase de apoio sem, no entanto, aumento da espasticidade [6].
A estimulação elétrica funcional (FES) é uma técnica de fortalecimento muscular que promove contração dos músculos privados de controle nervoso, com objetivos principais de aumentar a força muscular, reduzir a espasticidade, melhorar a amplitude de movimento (ADM), ativar os reflexos necessários à reorganização da atividade motora e possibilitar uma redução da assimetria cortical, pois o processamento sensório-motor durante a FES envolve processos relacionados à movimentação voluntária [7-9].

Diante dos dados expostos, cabe indagar quais os benefícios da FES na funcionalidade da marcha de crianças portadoras de PC após sua aplicação nos músculos tibial anterior e tríceps sural.

A motivação para desenvolver este estudo foi pela experiência com crianças portadoras de PC através do estágio extracurricular na APAE de Ubá/MG, além da associação da eletroestimulação no tratamento destas crianças.

Existem estudos que relatam os benefícios de um programa de fortalecimento em musculatura espástica em pacientes com AVE, mas não foi encontrada na literatura esta abordagem em pacientes com PC. Estudos já comprovaram que, além de reduzir a fraqueza muscular, o fortalecimento também aumenta a amplitude de movimento e reduz os valores de espasticidade. Essas alteraçóes atuam diretamente na funcionalidade por melhorar o padrão da marcha possibilitando a aquisição das habilidades diárias [8].

Este estudo é relevante para o reconhecimento da eletroestimulação como forma de tratamento em PC. Além de demonstrar os efeitos desta, quando aplicada no músculo tibial anterior e no músculo tríceps sural. Esta nova abordagem de tratamento seria válida para os acadêmicos de Fisioterapia assim como para os profissionais da área, pelo fato de apresentar efeitos positivos no tratamento da espasticidade. Os portadores de PC se beneficiariam com seus resultados permitindo melhor aquisição das atividades cotidianas.

Desta forma, o objetivo geral do presente estudo é comparar os efeitos da FES na funcionalidade da marcha em paciente com PC após sua aplicação nos músculos tibial anterior e tríceps sural. Os objetivos específicos constituem aumentar o grau de força muscular, aumentar a amplitude de movimento, reduzir o grau de espasticidade, aumentar velocidade e cadência da marcha, possibilitando uma melhor funcionalidade da marcha destes pacientes.

\section{Materiais e métodos}

Esta pesquisa é do tipo original com delineamento estudo de caso realizada na Associação de Pais e Amigos de Excepcionais (APAE) de Ubá/MG.

\section{Amostra}

Participaram da pesquisa dois pacientes de ambos os gêneros, com três anos de idade, selecionados intencionalmente, 
com o diagnóstico clínico de PC hemiparética e marcha independente.

\section{Instrumentos}

Foram mensuradas as amplitudes de movimentos (ADM) de dorsiflexão e plantiflexão do tornozelo do membro parético em decúbito dorsal por meio de goniômetro universal da marca CARCI, em movimentos ativo e passivo [10].

O teste manual de força muscular foi aplicado nos músculos tibial anterior e tríceps sural. Sua graduação baseia-se na habilidade de manter a parte testada em uma posição contra a gravidade, podendo ser classificado em: 0 - ausência de contração muscular; 1 - contração muscular, sem movimento articular; 2 - movimento articular sem atuação da gravidade; 3 - movimento contra a gravidade; 4 - movimento contra uma pequena resistência; 5 - movimento contra grande resistência [11].

O grau de espasticidade do músculo tríceps sural foi mensurado através da escala de Ashworth modificada, onde a articulação do tornozelo foi movimentada em dorsiflexão passiva de forma rápida e brusca, obtendo os resultados de acordo com a Tabela I [12].

Para análise da marcha foram avaliadas as variáveis velocidade, cadência, comprimento do passo, passada e largura da base de apoio, através do Protocolo de Cerny. Os instrumentos necessários foram: cronômetro da marca Herweg, uma passarela de 16 metros dispostos em 5 metros iniciais, 6 metros centrais e 5 metros finais (Figura 1). Os cinco metros iniciais e finais foram desconsiderados por representarem períodos de aceleração e desaceleração da marcha. $\mathrm{O}$ cronômetro foi acionado no momento que o paciente entrou na área central, a contagem foi interrompida quando o mesmo alcançou o final da área central [6].

Os cálculos foram extraídos a partir destes registros. Para marcar na passarela o comprimento dos passos, foi necessária a utilização de pó de café nas faces plantares. A colocação dos pés sobre o solo foi de maneira livre e espontânea, e a caminhada o mais rápido possível. Por convenção, os pacientes realizaram o protocolo descalços. Para cálculo do comprimento do passo e da passada foi calculado a média de três medidas aleatórias. O cálculo da velocidade foi realizado através da fórmula: $V=S / t^{6}$. A cadência foi calculada contando o número de passos por um minuto [5]. Para registro das imagens da marcha, antes e após a intervenção, utilizou-se filmadora da marca JVC, com autorização dos pais ou responsáveis.

As crianças foram submetidas à análise funcional utilizando o teste Gross Motor Function Measure (GMFM) para quantificar o desempenho motor. Este teste inclui 5 dimensões, porém foram analisadas apenas as dimensões: 4 - de pé; e 5 - andando, correndo e pulando, para tornar a pesquisa mais objetiva. Cada item foi mensurado pela observaçáo dos pacientes e classificados em uma escala ordinal de 4 pontos onde: $0=$ não faz; $1=$ inicia $(<10 \%) ; 2=$ completa parcialmente $(10 \mathrm{a}<100 \%) ; 3$ = completa a atividade [13].

Tabela I - Escala de Ashworth modificada [12].

\begin{tabular}{cl}
\hline Grau & \multicolumn{1}{c}{ Tônus muscular } \\
\hline 0 & Tônus normal muscular \\
1 & Leve aumento do tônus no final da amplitude de \\
& movimento. \\
$1+$ & Leve aumento de tônus na metade da ADM restante. \\
2 & Aumento marcante do tônus muscular durante a \\
& $\begin{array}{l}\text { maior parte da ADM. } \\
\text { Considerável aumento do tônus. Movimento passivo }\end{array}$ \\
& difícil de realizar. \\
& Rigidez em flexão ou extensão
\end{tabular}

\section{Procedimento}

As avaliaçóes utilizando os instrumentos citados anteriormente foram realizadas antes e após a intervenção fisioterapêutica. Foi sorteado o local para a eletroestimulação. No paciente 1 , os eletrodos de borracha, tamanho $5 \times 5$ centímetros $(\mathrm{cm})$, foram posicionados um no ponto motor do músculo tibial anterior e outro $2 \mathrm{~cm}$ abaixo. Durante a passagem da corrente foi solicitado o movimento de subir degraus (15 $\mathrm{cm}$ ). Na paciente 2, os eletrodos foram posicionados um no ponto motor do músculo tríceps sural e outro $2 \mathrm{~cm}$ abaixo; na passagem da corrente realizou-se o movimento de descer degraus $(15 \mathrm{~cm})$ [5].

Foi utilizado o aparelho Physiotonus Four da marca Bioset, com aplicação de correntes de eletroestimulação neuromuscular através da corrente de baixa frequência bifásica FES, com parâmetros fixos em $250 \mu$ s modulados a $50 \mathrm{~Hz}$, Ton 06 seg, Toff 12 seg, e intensidade conforme a tolerância do paciente. Foram realizadas sessóes com duração de 20 minutos, três vezes por semana em dias intercalados, durante sete semanas, totalizando 21 sessóes. Durante a pesquisa os pacientes não foram submetidos à fisioterapia convencional [13].

O projeto de pesquisa foi encaminhado ao Comitê de ética em Pesquisa da Unipac obedecendo à resoluçáo do Conselho Nacional de Saúde (CNS) 196/96.

\section{Análise estatistica}

Foi realizada uma análise descritiva dos dados de força e tônus muscular, ADM e o escore da escala GMFM.

\section{Resultados e discussão}

Após a eletroestimulação foi observada melhora da atividade funcional da marcha avaliada pelas dimensões D (em pé) e E (andar, correr, pular) da escala GMFM, nas duas crianças, porém esta foi maior naquela que recebeu eletroestimulação no músculo tríceps sural (Figura 1/ Tabela II e III). 
Tabela II - Apresentação dos resultados da escala GMFM no paciente 1 (tibial anterior).

\begin{tabular}{cccc}
\hline GMFM (Pac 1) & Pré-eletro & Pós-eletro & Evolução \\
\hline Dimensão D (39) & 21 & 36 & 15 \\
Dimensão E (72) & 42 & 55 & 13 \\
\hline
\end{tabular}

Tabela III - Apresentação dos resultados da escala GMFM no paciente 2 (triceps sural).

\begin{tabular}{cccc}
\hline GMFM (Pac 2) & Pré-eletro & Pós-eletro & Evolução \\
\hline Dimensão D (39) & 16 & 34 & 18 \\
Dimensão E (72) & 29 & 45 & 16 \\
\hline
\end{tabular}

Figura 1 - Apresentação dos resultados das dimensöes $D$ e $E$ (GMFM) em porcentagem nos pacientes 1 e 2 . GMFM

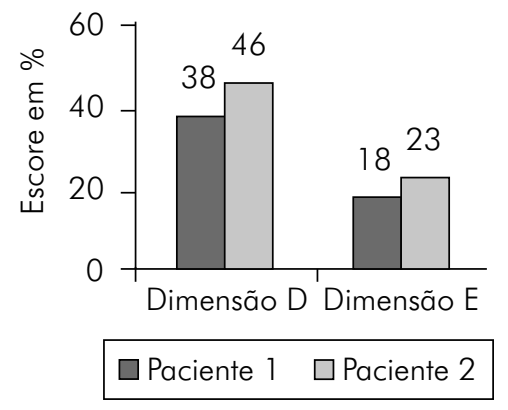

O estudo de Drouin et al. [14] concluiu que as dimensóes D e E do GMFM podem ser usadas como prognóstico locomotor. Complementando este estudo, Damiano et al. [15] investigaram a correlação entre o GMFM e os parâmetros da marcha em crianças com PC espástica, e confirmaram que a deambulação é representativa do status motor global na PC, e que o GMFM e a análise da marcha são medidas complementares na avaliaçáo funcional destas crianças.

Foram observadas melhora na ADM ativa de plantiflexão no Pac 1 de $10^{\circ}$, no $\operatorname{Pac} 2$ de $25^{\circ}$, mantiveram-se as medidas de dorsiflexão ativa nos dois pacientes. A evolução da $\mathrm{ADM}$ passiva foi maior no $\operatorname{Pac} 2$, cerca de $10^{\circ}$ de plantiflexão e $20^{\circ}$ de dorsiflexão. O Pac 1 obteve $10^{\circ}$ a mais na ADM passiva de plantiflexão e a dorsiflexão se manteve (Tabela IV e V).

Tabela IV - Resultados obtidos da ADM ativa antes e após tratamento.

\begin{tabular}{ccc}
\hline ADM ativa & Pac 1 & Pac 2 \\
\hline Plantiflexão & $>10^{\circ}$ & $>25^{\circ}$ \\
\hline
\end{tabular}

Tabela V - Resultados obtidos da ADM ativa antes e após tratamento.

\begin{tabular}{lcc}
\hline \multicolumn{1}{c}{ ADM passiva } & Pac 1 & Pac 2 \\
\hline Plantiflexão & $>10^{\circ}$ & $>10^{\circ}$ \\
Dorsiflexão & $-\cdots--$ & $>20^{\circ}$ \\
\hline
\end{tabular}

Os resultados obtidos nos pacientes 1 e 2 no movimento de dorsiflexão ativa não houve diferença significativa, assim como o movimento de dorsiflexão passiva no paciente 1 .
Arantes et al. [7] concluíram através de uma revisão da literatura que não existem ganhos significativos da $\mathrm{ADM}$ após eletroestimulação. Já Martins et al. [16] puderam concluir que a FES é um recurso coadjuvante terapêutico importante para obtenção da movimentação tanto ativa quanto passiva.

Em relaçáo ao tônus muscular houve uma redução do grau na escala Ashworth no Pac 2 e manutenção do grau no Pac 1(Tabela VI).

Tabela VI - Avaliação do tônus muscular de tríceps sural dos pacientes pré e pós eletroestimulação.

\begin{tabular}{lll}
\hline Escala Ashworth & \multicolumn{1}{c}{ Pré } & \multicolumn{1}{c}{ Pós } \\
\hline Pac 1 & $1+:$ Leve aumento & $1+:$ Leve aumento \\
& de tônus na metade & de tônus na metade \\
& da ADM restante & da ADM restante. \\
Pac 2 & $1+:$ Leve aumento & $1:$ Leve aumento de \\
& de tônus na metade tônus no final da \\
& da ADM restante & ADM \\
\hline
\end{tabular}

Arantes et al. [7] corroboram estes resultados com Junqueira et al. [11] e Salmela-Teixeira $e t$ al. [17] quando concluem que técnicas de fortalecimento não aumentam o tônus muscular, podendo até reduzir seus valores. Guimarães et al. [6] constataram manutenção do tônus muscular após fortalecimento de musculatura espástica. Wang et al. [18] afirmam que a eletroestimulação promove redução da espasticidade em decorrência da coativação dos motoneurônios alfa e gama dos músculos paréticos [18].

Hamill et al. [19] afirmam que o músculo espástico é considerado um músculo encurtado e incapaz de gerar muita tensão contrátil porque seus filamentos protéicos ficam sobrepostos criando uma ativaçáo incompleta das pontes cruzadas. Assim, a diminuição de pontes cruzadas desenvolve um músculo fraco e inadequado para gerar grandes quantidades de força. Desta forma, a fraqueza muscular reflete a incapacidade de gerar força pela diminuição da ativação das unidades motoras ou das mudanças fisiológicas do músculo parético.

Outro achado relevante deste estudo foi que o grau de força muscular de plantiflexores do paciente 1 diminuiu, enquanto que do paciente 2 aumentou. A força muscular de dorsiflexores se manteve no paciente 1 e aumentou no paciente 2. (Figura 2 e 3 ).

Figura 2 - Força muscular de plantiflexores pré e pós eletroestimulação.

GMFM

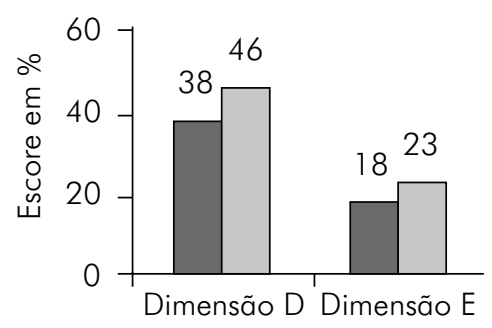

Paciente $1 \quad \square$ Paciente 2 
Figura 3 - Força muscular de dorsiflexores pré e pós eletroestimulação.

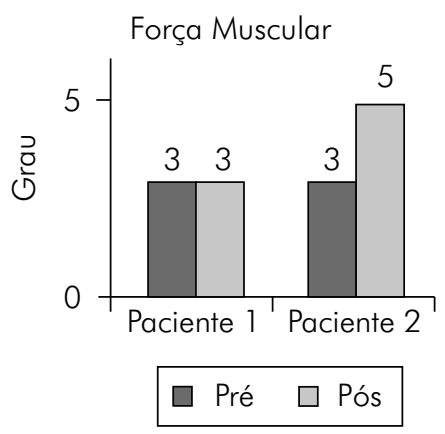

Corroborando com os resultados obtidos, Guimarães et al. [6] ainda encontraram em seu estudo melhora significativa da força muscular após a eletroestimulação [6]. Junqueira [11] afirma que técnicas e exercícios de fortalecimento muscular atuam aumentando o recrutamento de unidades motoras, melhorando a capacidade de geração de força, reduzindo a hiperativação reflexa e preservando a extensibilidade funcional dos músculos.

As variáveis da marcha avaliadas através do protocolo de Cerny demonstraram melhoras nos dois pacientes (Tabela VII e VIII).

Tabela VII - Apresentação dos resultados do protocolo de Cerny no paciente 1 após aplicação da FES.

\begin{tabular}{lccc}
\hline & \multicolumn{3}{c}{ Paciente 1 } \\
\hline $\begin{array}{l}\text { Protocolo } \\
\text { de Cerny }\end{array}$ & Antes & Depois & Evolução \\
\hline $\begin{array}{l}\text { Tempo da } \\
\text { área central }\end{array}$ & $7 \mathrm{seg}$ & $5 \mathrm{seg}$ & $<2 \mathrm{seg}$ \\
$\begin{array}{l}\text { Média dos } \\
\text { passos }\end{array}$ & $21 \mathrm{~cm}$ & $29 \mathrm{~cm}$ & $>8 \mathrm{~cm}$ \\
$\begin{array}{l}\text { Passada } \\
\text { Largura da }\end{array}$ & $50 \mathrm{~cm}$ & $57 \mathrm{~cm}$ & $>7 \mathrm{~cm}$ \\
base de & $22 \mathrm{~cm}$ & $14 \mathrm{~cm}$ & $<8 \mathrm{~cm}$ \\
apoio & & & \\
$\begin{array}{l}\text { Velocidade } \\
\text { Cadência }\end{array}$ & $0,86 \mathrm{~m} / \mathrm{seg}$ & $1,2 \mathrm{~m} / \mathrm{seg}$ & $>0,34 \mathrm{~m} / \mathrm{seg}$ \\
& & & $<12 \mathrm{passo} / \mathrm{min}$ \\
\hline
\end{tabular}

Para crianças saudáveis na faixa etária dos 4 anos de idade, os valores médios encontrados para o comprimento da passada, cadência e velocidade são $0,78 \mathrm{~cm}, 152$ passos $/ \mathrm{min}$ e $0,99 \mathrm{~m} / \mathrm{seg}$, respectivamente. Jerônimo et al. [5] obtiveram aumento da velocidade e redução da cadência após aplicação da FES em tibial anterior. Shuster et al. [12] afirmam que esses resultados só são obtidos porque a eletroestimulação promove um feedback cutâneo e aumento da propriocepçáo, melhorando o equilíbrio e favorecendo a marcha.

Damázio et al. [20] relatam que o melhor desempenho no protocolo de Cerny pode ser devido a melhora da ADM, da ativação de placas motoras pela FES e da redução do tônus muscular. Desta forma, a FES tem demonstrado ser um protocolo confiável, uma vez que promove recrutamento neuronal em áreas seletas, na mesma quantidade em cada hemisfério cerebral reduzindo a assimetria cortical $[21,22]$.

Tabela VIII - Apresentação dos resultados do protocolo de Cerny no paciente 2 após aplicação da FES.

\begin{tabular}{|c|c|c|c|}
\hline & \multicolumn{3}{|c|}{ Paciente 2} \\
\hline $\begin{array}{l}\text { Protocolo } \\
\text { de Cerny }\end{array}$ & Antes & Depois & Evolução \\
\hline $\begin{array}{l}\text { Tempo da } \\
\text { área central }\end{array}$ & $15 \mathrm{seg}$ & $6 \mathrm{seg}$ & $<10 \mathrm{seg}$ \\
\hline $\begin{array}{l}\text { Média dos } \\
\text { passos }\end{array}$ & $23 \mathrm{~cm}$ & $31 \mathrm{~cm}$ & $>8 \mathrm{~cm}$ \\
\hline Passada & $49 \mathrm{~cm}$ & $74 \mathrm{~cm}$ & $>25 c$ \\
\hline $\begin{array}{l}\text { Largura da } \\
\text { base de }\end{array}$ & $15 \mathrm{~cm}$ & $09 \mathrm{~cm}$ & $<6 \mathrm{~cm}$ \\
\hline apoio & & & \\
\hline Velocidade & $0,4 \mathrm{~m} / \mathrm{seg}$ & $1,0 \mathrm{~m} / \mathrm{seg}$ & $>0,6 \mathrm{~m} / \mathrm{seg}$ \\
\hline Cadência & $112 \mathrm{pas} / \mathrm{min}$ & $99 \mathrm{pas} / \mathrm{min}$ & $\begin{array}{l}<12 \text { passo/ } \\
\min \end{array}$ \\
\hline
\end{tabular}

\section{Conclusão}

Os resultados obtidos encontraram efeitos mais satisfatórios na criança com PC submetida à FES em tríceps sural, pois aumentou a força muscular, os padróes da marcha evoluíram, houve redução do tônus muscular, aumento da $\mathrm{ADM}$ de plantiflexão e dorsiflexão em movimentos ativos e passivos, após 21 sessóes. Ao contrário do que é frequentemente utilizado no tratamento de pacientes com espasticidade, o fortalecimento do músculo espástico demonstrou resultados positivos. Desta forma recomenda-se a seleção de uma amostra aleatória com uma populaçáo maior de pacientes portadores de PC do tipo hemiparéticos espásticos, assim como a realização de um grupo controle, para determinar com maior fidedignidade os benefícios da FES.

\section{Referências}

1. Maranhão MVM. Anestehesia and cerebral palsy. Rev Bras Anestesiol 2005;55(6):680-702.

2. Vivone GP, Tavares MMM, Bartolomeu RS, Nemr K, Chiappetta ALM. Análise da consistência alimentar e tempo de deglutição em crianças com paralisia cerebral tetraplégica espástica. Rev CEFAC 2007;9(4):504-11.

3. Rotta NT. Paralisia cerebral, novas perspectivas terapêuticas. J Pediatr 2002;78(1):48-54.

4. Franco CB, Pires LC, Pontes LS, Souza EJ. Avaliação da amplitude de movimento do tornozelo em crianças com paralisia cerebral após aplicação de toxina botulínica seguida de fisioterapia. Rev Para Med 2006;20(3):43-9.

5. Jerônimo BP, Borges MBS, Dini PD, David AC. Variáveis espaço-temporais da marcha de crianças com paralisia cerebral submetidas a eletroestimulação no músculo tibial anterior. Rev Bras Fisioter 2007;11(4):261-6. 
6. Guimarães RM, Pereira JS, Batista LA. Fortalecimento do músculo sóleo: impacto na cinemática da marcha de indivíduos hemiparéticos. Fisioter Mov 2007;20(3):11-6.

7. Arantes NF, Vaz DV, Mancini MC, Pereira MSDC, Pinto FP, Pinto TPS. Efeitos da estimulação elétrica funcional nos músculos do punho e dedos em indivíduos hemiparéticos: uma revisão sistemática da literatura. Rev Bras Fisioter 2007;11(6):419-27.

8. Ecard L, Silva APS, Neto MP, Veiga H, Cagy M, Piedade et al. Os efeitos da estimulação elétrica funcional na assimetria cortical inter-hemisférica. Arq Neuropsiquiatr 2007;65(3):642-6.

9. Ecard L, Silva APS, Neto MP, Veiga H, Cagy M, Piedade R et al. Alteraçóes na coerência cortical inter-hemisférica produzidas pela estimulaçáo elétrica funcional (FES). Arq Neuropsiquiatr 2007;65(2-A):327-31.

10. Venturini C, Ituassú NT, Teixeira LM, Deus CVO. Confiabilidade intra e interexaminadores de dois métodos de medidas da amplitude ativa de dorsiflexão do tornozelo. Rev Bras Fisioter 2006;10(4):407-11.

11. Junqueira RT, Ribeiro AMB, Scianni AA. Efeitos do fortalecimento muscular e sua relação com a atividade funcional e a espasticidade em indivíduos hemiparéticos. Rev Bras Fisioter 2004;8(3):247-52.

12. Schuster RC, Sant CR, Dalbosco V. Efeitos da estimulação elétrica funcional (FES) sobre o padráo de marcha de um paciente hemiparético. Acta Fisiatr 2007;14(2):82-6.

13. Calcágno NC, Pinto TPS,Vaz DV, Mancini MC, Sampaio RF. Análise dos efeitos da utilização de tala seriada em crianças portadoras de paralisia cerebral: uma revisão sistemática da literatura. Rev Bras Saude Matern Infant 2006;6(1):11-22.
14. Drouin LM, Malouin F, Richard CL, Marcoux S. Correlation between the gross motor function measure scores and gait spatiotemporal measures in children with neurological impairments. Dev Med Child Neurol 1996;38:1007-19.

15. Damiano DL, Abel MF. Relation of gait analysis to gross motor function in cerebral palsy. Dev Med Child Neurol 1996;38:389-96.

16. Martins FLM, Guimarães LHCT, Vitorino DFM, Souza LCF. Eficácia da eletroestimulação funcional na amplitude de movimento de dorsiflexão de hemiparéticos. Neurociências 2004;12(2):1-7.

17. Salmela-Teixeira LF, Only SJ, Nadeau S, Brouwer B. Muscle strengthening and physical conditioning to reduce impairment and disability in chronic stroke survivors. Arch Phys Med Rehabil 1999;80:1211-8.

18. Wang RY, Tsai MW, Chan RC. Effects of surface spinal cord stimulation on spasticity and quantitative assessment of muscle tone in hemiplegic patients. Am J Phys Med Rehabil 1988;77(4):282-7.

19. Hamill J, Knutzen KM. Bases biomecânicas do movimento humano. São Paulo: Manole; 1999.

20. Damázio LCM, Carvalho JAG, Assis LR, Correia F, Ribeiro H. Biomechanical gait analysis of hemiparetic patients after functional electric stimulation application. Motor Control Journal 2007;S11:220.

21. Brasileiro JS, Salvini TS. Limites da estimulação elétrica funcional no fortalecimento de músculos com déficit de força. Fisioter Bras 2004;5(3):224-30.

22. Kitchens BS. Eletroterapia de Clayton. $10^{\text {a }}$ ed. São Paulo: Manole; 1998. 